\title{
MIXED VECTOR FQ-IMPLICIT VARIATIONAL INEQUALITY WITH LOCAL NON-POSITIVITY
}

\author{
Byung-SOO LeE
}

\begin{abstract}
This paper introduces a local non-positivity of two set-valued mappings $(F, Q)$ and considers the existences and properties of solutions for set-valued mixed vector $F Q$-implicit variational inequality problems and set-valued mixed vector $F Q$-complementarity problems in the neighborhood of a point belonging to an underlined domain $K$ of the set-valued mappings, where the neighborhood is contained in $K$.

This paper generalizes and extends many results in [1, 3-7].
\end{abstract}

\section{Introduction}

$F$-complementarity problem (F-CP); finding $x \in K$ such that

$$
\langle T x, x\rangle+F(x)=0 \text { and }\langle T x, y\rangle+F(y) \geq 0 \text { for all } y \in K,
$$

and corresponding variational inequality problem;

finding $x \in K$ such that

$$
\langle T x, y-x\rangle+F(y)-F(x) \geq 0 \text { for all } y \in K,
$$

where $K$ is a nonempty closed and convex cone of a real Banach space $X$ with its dual $X^{*}, T: K \rightarrow X^{\star}$ is a mapping and $F: K \rightarrow(-\infty,+\infty)$ is a positively homogeneous and convex function, were firstly considered in [7].

In 2003, Fang and Huang [1] considered a vector $F$-complementarity problem with demi-pseudomonotone mappings in Banach spaces by considering the solvability of the problems. Huang and Li [3] studied a scalar F-implicit variational inequality problem and another $F$-implicit complementarity problem in Banach spaces in 2004. Recently, the result of the scalar case in [3] was extended and generalized to the vector case by $\mathrm{Li}$ and Huang [6] . The equivalence between the $F$-implicit variational inequality problem and $F$-implicit complementarity problem was presented and some new existence theorems of solutions for $F$-implicit variational inequality problems were also proved.

Received December 23, 2008.

2000 Mathematics Subject Classification. 90C33, 49J40.

Key words and phrases. mixed vector $F Q$-implicit complementarity problem, mixed vector $F Q$-implicit variational inequality problem, positively homogeneous mapping, convex cone, upper semicontinuity, lower semicontinuity, locally non-positive. 
In 2007, Lee, Khan, and Salahuddin [5] generalized some results of $[3,6]$ to more generalized vector case. They introduced a generalized vector $F$-implicit complementarity problem and corresponding generalized vector $F$-implicit variational inequality problem in Banach spaces and proved the equivalence between them under certain assumptions. Furthermore, they derived some new existence theorems of solutions for the generalized vector $F$-implicit complementarity problems and the generalized vector $F$-implicit variational inequality problems under some suitable assumptions without any monotonicity.

Recently, the following mixed vector $F Q$-implicit variational inequality problem (FQ-VI) and corresponding mixed vector $F Q$-implicit complementarity problems (FQ-CP) for set-valued mappings were considered in [4];

(FQ-VI); find $x \in K$ such that $p-s+w-z \in P(x)$ for any $p \in Q(x, g(y))$, $s \in Q(x, h(x)), w \in F(g(y))$, and $z \in F(h(x))$, where $y \in K$.

(FQ-CP); find $x \in K$ such that

(a) $p+w \in P(x)$ for any $p \in Q(x, g(y))$ and $w \in F(g(y))$, where $y \in K$, and

(b) $s+z=0$ for any $s \in Q(x, h(x))$ and $z \in F(h(x))$, where $K$ is a nonempty closed convex cone of a real Banach space $X$ and $\{P(x)$ : $x \in K\}$ is a family of nonempty pointed closed convex cones with the apex at the origin in a real Banach space $Y$. Mappings $g, h: K \rightarrow K$ are single-valued, $F: K \rightarrow 2^{Y}$ and $Q: K \times K \rightarrow 2^{Y}$ are set-valued.

The following Theorem A and Theorem B in [4] show the equivalence between (FQ-VI) and (FQ-CP) and some existence theorems of solutions for them under some suitable assumptions without monotonicity, respectively.

Theorem A. Assume that a set-valued mapping $F: K \rightarrow 2^{Y}$ is positively homogeneous, a set-valued mapping $Q: K \times K \rightarrow 2^{Y}$ is also positively homogeneous in the second argument and $g: K \rightarrow K$ is surjective. Then (FQ-VI) is equivalent to $(F Q-C P)$.

Theorem B. Let $K$ be a nonempty closed convex subset of $X$ and $P: K \rightarrow 2^{Y}$ be upper semicontinuous on $K$. Assume that

(a) $g, h: K \rightarrow K$ are continuous, $F: K \rightarrow 2^{Y}$ is lower semicontinuous and $Q: K \times K \rightarrow 2^{Y}$ is lower semicontinuous in two arguments,

(b) there exists a single-valued mapping $T: K \times K \rightarrow Y$ satisfying

(b1) for $x \in K, T(x, x) \in P(x)$,

(b2) for $x, y \in K$,

$$
a-b+c-d-T(x, y) \in P(x)
$$

for any $a \in Q(x, g(y)), b \in Q(x, h(x)), c \in F(g(y))$ and $d \in$ $F(h(x))$,

(b3) for $x \in K$ the set $\{y \in K: T(x, y) \notin P(x)\}$ is convex, 
(c) there exists a nonempty compact convex subset $D$ of $K$ such that for all $x \in K \backslash D$ there exists a $y \in D$ satisfying $a-b+c-d \notin P(x)$ for some $a \in Q(x, g(y)), b \in Q(x, h(x)), c \in F(g(y))$ and $d \in F(h(x))$.

Then $(F Q-V I)$ has a solution. Furthermore, the solution set of $(F Q-V I)$ is closed.

This paper introduces a local non-positivity of set-valued mappings $(F, Q)$ and considers the existences and properties of solutions for (FQ-VI) and (FQ$\mathrm{CP}$ ) in the neighborhood of a point belonging to an underlined domain $K$ of the set-valued mappings, where the neighborhood is contained in $K$.

This paper generalizes and extends many results in $[1,3-7]$.

\section{Preliminaries}

Remark that $P(x), x \in K$ is a closed set such that

(i) $\lambda P(x) \subset P(x), \lambda>0, x \in K$,

(ii) $P(x)+P(x) \subset P(x), x \in K$,

(iii) $P(x) \cap(-P(x))=\{0\}, x \in K$.

An ordered Banach space $(Y, P(x))$ is a real Banach space with an ordering defined by a closed cone $P(x) \subset Y$ as for any $y, z \in Y$,

$$
\begin{array}{lll}
y \geq z & \text { if and only if } & y-z \in P(x), \\
y \geq z & \text { if and only if } & y-z \notin P(x) .
\end{array}
$$

Remark that

$$
\begin{aligned}
& z \leq 0 \quad \text { if and only if } \quad z \in-P(x), \\
& z \not \leq 0 \text { if and only if } \quad z \notin-P(x), \\
& z \geq 0 \quad \text { if and only if } \quad z \in P(x), \\
& z \nsupseteq 0 \quad \text { if and only if } \quad z \notin P(x) .
\end{aligned}
$$

Lemma $2.1([1])$. Let $(Y, P)$ be an ordered Banach space induced by a pointed closed cone $P$. Then $x+y \in P$ for $x, y \in P$.

Definition 2.1 ([4]). Let $X, Y$ be two vector spaces and $K$ be a cone of $X$. A set-valued mapping $F: K \rightarrow 2^{Y}$ is said to be positively homogeneous if $F(\alpha x)=\alpha F(x)$ for all $x \in K$ and $\alpha \geq 0 . F$ is said to be linear if $F(\alpha x+\beta y)=$ $\alpha F(x)+\beta F(y)$ for $x, y \in K, \alpha+\beta=1, \alpha, \beta \geq 0$.

Definition 2.2. A set-valued mapping $W: K \subset X \rightarrow 2^{Y}$ is upper semicontinuous at $x_{0} \in K$ if every open set $V$ containing $W\left(x_{0}\right)$ there exists an open set $U$ containing $x_{0}$ such that $W(U) \subset V$. W is lower semicontinuous at $x_{0} \in K$ if for every open set $V$ intersecting $W\left(x_{0}\right)$ there exists an open set $U$ containing $x_{0}$ such that $W(x) \cap V \neq \emptyset$ for every $x \in U$. $W$ is upper semicontinuous (lower semicontinuous) on $K$ if it is upper semicontinuous (lower semicontinuous) at every point of $K . W$ is continuous on $K$ if it is both upper semicontinuous and lower semicontinuous on $K$. 
Lemma 2.2. Let $W: X \rightarrow 2^{Y}$ be a set-valued mapping and $x_{0} \in X$.

(i) $W$ is upper semicontinuous at $x_{0}$ if and only if for any net $\left\{x_{\alpha}\right\} \subset X$ with $x_{\alpha} \rightarrow x_{0}$ and for any net $\left\{y_{\alpha}\right\}$ in $Y$ with $y_{\alpha} \in W\left(x_{\alpha}\right)$ such that $y_{\alpha} \rightarrow y_{0}$ in $Y$, we have $y_{0} \in W\left(x_{0}\right)$.

(ii) $W$ is lower semicontinuous at $x_{0}$ if and only if for any net $\left\{x_{\alpha}\right\} \subset X$ with $x_{\alpha} \rightarrow x_{0}$, and for any $y_{0} \in W\left(x_{0}\right)$, there exists a net $\left\{y_{\alpha}\right\}$ such that $y_{\alpha} \in W\left(x_{\alpha}\right)$ and $y_{\alpha} \rightarrow y_{0}$.

Lemma $2.3([2])$. Let $W: X \rightarrow 2^{Y}$ be a set-valued mapping. If for any $x \in X$, $W(x)$ is compact, then $W$ is upper semicontinuous at $x_{0}$ if and only if for any net $\left\{x_{\alpha}\right\} \subset X$ such that $x_{\alpha} \rightarrow x_{0}$ and for every $y_{\alpha} \in W\left(x_{\alpha}\right)$, there exists $y_{0} \in W\left(x_{0}\right)$ and a subnet $\left\{y_{\alpha_{\beta}}\right\}$ of $\left\{y_{\alpha}\right\}$ such that $y_{\alpha_{\beta}} \rightarrow y_{0}$.

\section{Main results}

Unless otherwise specified, we assume that $K$ is a nonempty closed convex cone of a real Banach space $X$ and $\{P(x): x \in K\}$ is a family of nonempty pointed closed convex cones with the apex at the origin in a real Banach space $Y$.

Definition 3.1. Let $g, h: K \rightarrow K$ be single-valued mappings and $F: K \rightarrow 2^{Y}$, $Q: K \times K \rightarrow 2^{Y}$ set-valued mappings. Let $P: K \rightarrow 2^{Y}$ be a set-valued mapping with nonempty pointed closed convex cones with the apex at the origin in $Y .(F, Q)$ is said to be locally non-positive at $x_{0} \in K$ with respect to $(g, h)$ if there exist a neighborhood $N\left(x_{0}\right)$ of $x_{0}$ and $z_{0} \in K \cap \operatorname{Int} N\left(x_{0}\right)$ such that $a-b+c-d \in-P(x)$ for any $a \in Q\left(x, g\left(z_{0}\right)\right), b \in Q(x, h(x)), c \in F\left(g\left(z_{0}\right)\right)$ and $d \in F(h(x))$ for $x \in K \cap \partial N\left(x_{0}\right)$, the boundary of $N\left(x_{0}\right)$.

Example 3.1. Let $X=Y=\mathbb{R}, K=[0, \infty)$ and $P(x)=[0, \infty)$ for all $x \in K$. Define mappings $g, h: K \rightarrow K$ by $g(x)=2 x$ and $h(x)=2 x$, set-valued mappings $F: K \rightarrow 2^{\mathbb{R}}$ by $F(x)=\left[\frac{1}{2} x, x\right], Q: K \times K \rightarrow 2^{\mathbb{R}}$ by $Q(x, y)=\left[\frac{2}{3}(x+y), x+y\right]$, then $(F, Q)$ is locally non-positive at $x_{0}=0 \in K$ with respect to $(g, h)$. If we take a neighborhood $N(0)=\left(-\frac{1}{2}, \frac{1}{2}\right)$ of $x_{0}=0$ and $z_{0}=\frac{1}{4} \in K \cap \operatorname{Int} N(0)=\left[0, \frac{1}{2}\right)$, then for the unique element $x=\frac{1}{2}$ of $K \cap \partial N(0)=\left\{\frac{1}{2}\right\}$, we have for any $a \in Q\left(\frac{1}{2}, g\left(\frac{1}{4}\right)\right), b \in Q\left(\frac{1}{2}, h\left(\frac{1}{2}\right)\right)$, $c \in F\left(g\left(\frac{1}{4}\right)\right)$ and $d \in F\left(h\left(\frac{1}{2}\right)\right)$,

$$
a-b+c-d \in-K \text {. }
$$

In fact, $Q\left(\frac{1}{2}, g\left(\frac{1}{4}\right)\right)=Q\left(\frac{1}{2}, \frac{1}{2}\right)=\left[\frac{2}{3}, 1\right], Q\left(\frac{1}{2}, h\left(\frac{1}{2}\right)\right)=Q\left(\frac{1}{2}, 1\right)=\left[1, \frac{3}{2}\right]$, $F\left(g\left(\frac{1}{4}\right)\right)=F\left(\frac{1}{2}\right)=\left[\frac{1}{4}, \frac{1}{2}\right], F\left(h\left(\frac{1}{2}\right)\right)=F(1)=\left[\frac{1}{2}, 1\right]$, thus

$$
1-1+\frac{1}{2}-\frac{1}{2}=0 \in-K \text {. }
$$

Theorem 3.1. Let $K$ be a nonempty closed and convex subset of $X$. Let $P: K \rightarrow 2^{Y}$ be a set-valued mapping with nonempty pointed closed convex cones with the apex at the origin in $Y$. Assume that 
(a) single-valued mappings $g, h: K \rightarrow K$ are continuous and set-valued mappings $F: K \rightarrow 2^{Y}, Q: K \times K \rightarrow 2^{Y}$ are continuous and $P$ is upper semicontinuous,

(b) a single-valued mapping $T: K \times K \rightarrow Y$ satisfies

(b1) for $x \in K, T(x, x) \in P(x)$,

(b2) for $x, y \in K$,

$$
\begin{aligned}
& \qquad a-b+c-d-T(x, y) \in P(x) \\
& \text { for any } a \in Q(x, g(y)), b \in Q(x, h(x)), c \in F(g(y)) \text { and } d \in \\
& F(h(x)), \\
& \text { (b3) for } x \in K \text { the set }\{y \in K: T(x, y) \notin P(x)\} \text { is convex, }
\end{aligned}
$$

(c) $(F, Q)$ is locally non-positive at $x_{0} \in K$ with respect to $(g, h)$ and there exists a nonempty compact convex subset $D$ of $K \cap N\left(x_{0}\right)$ such that for all $x \in\left(K \cap N\left(x_{0}\right)\right) \backslash D$ there exists $y \in D$ satisfying

$$
a-b+c-d \notin P(x)
$$

for any $a \in Q(x, g(y)), b \in Q(x, h(x)), c \in F(g(y))$ and $d \in F(h(x))$,

(d) $g, h$ and $F$ are linear and $Q$ is linear in the second argument.

Then (FQ-VI) has a solution in the neighborhood of $x_{0}$, that is, there exists $x^{*} \in K \cap N\left(x_{0}\right)$ such that, for $y \in K$

$$
a^{*}-b^{*}+c-d^{*} \in P\left(x^{*}\right)
$$

for any $a^{*} \in Q\left(x^{*}, g(y)\right), b^{*} \in Q\left(x^{*}, h\left(x^{*}\right)\right), c \in F(g(y))$ and $d^{*} \in F\left(h\left(x^{*}\right)\right)$.

Proof. Since $(F, Q)$ is locally non-positive at $x_{0} \in K$ with respect to $(g, h)$, we can assume that $N\left(x_{0}\right)$ is a closed and convex set without loss of generality. Since $K \cap N\left(x_{0}\right)$ is also closed and convex, from Theorem B, (FQ-VI) has a solution $x^{*} \in K \cap N\left(x_{0}\right)$ such that, for $y \in K \cap N\left(x_{0}\right)$

$$
a^{*}-b^{*}+c-d^{*} \in P\left(x^{*}\right)
$$

for any $a^{*} \in Q\left(x^{*}, g(y)\right), b^{*} \in Q\left(x^{*}, h\left(x^{*}\right)\right), c \in F(g(y))$ and $d^{*} \in F\left(h\left(x^{*}\right)\right)$.

Now we show that for $y \in K,(3.1)$ also holds.

(i) If $x^{*} \in K \cap \operatorname{Int} N\left(x_{0}\right)$, then $N\left(x_{0}\right) \backslash\left\{x^{*}\right\}$ is a neighborhood of the origin and so it is absorbing. For any $y \in K$, there exists $t \in(0,1)$ such that $t\left(y-x^{*}\right) \in$ $N\left(x_{0}\right) \backslash\left\{x^{*}\right\}$ and so $y_{t}:=t y+(1-t) x^{*} \in K \cap N\left(x_{0}\right)$. Hence

$$
a_{t}^{*}-b^{*}+c_{t}-d^{*} \in P\left(x^{*}\right)
$$

for any $a_{t}^{*} \in Q\left(x^{*}, g\left(y_{t}\right)\right), b^{*} \in Q\left(x^{*}, h\left(x^{*}\right)\right), c_{t} \in F\left(g\left(y_{t}\right)\right)$ and $d^{*} \in F\left(h\left(x^{*}\right)\right)$.

On the other hand, the following set

$$
\begin{gathered}
A=\{y \in K: a-b+c-d \in P(x) \text { for any } a \in Q(x, g(y)), b \in Q(x, h(x)) \\
c \in F(g(y)) \text { and } d \in F(h(x))\},
\end{gathered}
$$

is convex for all $x \in K$. In fact, if $y_{1}, y_{2} \in A$, then for $x \in K$,

$$
a_{1}-b+c_{1}-d \in P(x)
$$


for any $a_{1} \in Q\left(x, g\left(y_{1}\right)\right), b \in Q(x, h(x)), c_{1} \in F\left(g\left(y_{1}\right)\right)$ and $d \in F(h(x))$ and

$$
a_{2}-b+c_{2}-d \in P(x)
$$

for any $a_{2} \in Q\left(x, g\left(y_{2}\right)\right), b \in Q(x, h(x)), c_{2} \in F\left(g\left(y_{2}\right)\right)$ and $d \in F(h(x))$. Hence for $t \in(0,1)$, from the condition (d), we have, for $x \in K$

$$
\begin{aligned}
& t a_{1}+(1-t) a_{2}-b+t c_{1}+(1-t) c_{2}-d \in P(x) \\
& \text { for any } t a_{1}+(1-t) a_{2} \in t Q\left(x, g\left(y_{1}\right)\right)+(1-t) Q\left(x, g\left(y_{2}\right)\right) \\
& =Q\left(x, g\left(t y_{1}+(1-t) y_{2}\right)\right) \text {, } \\
& b \in Q(x, h(x)) \text {, } \\
& t c_{1}+(1-t) c_{2} \in t F\left(g\left(y_{1}\right)\right)+(1-t) F\left(g\left(y_{2}\right)\right) \\
& =F\left(g\left(t y_{1}+(1-t) y_{2}\right)\right), \text { and } \\
& d \in F(h(x)) .
\end{aligned}
$$

Hence $t y_{1}+(1-t) y_{2} \in A$, which shows that $A$ is convex. Thus by the continuities of $g, h, F$ and $Q$ from (3.2) we have for $y \in K$

$$
a^{*}-b^{*}+c-d^{*} \in P\left(x^{*}\right)
$$

for any $a^{*} \in Q\left(x^{*}, g(y)\right), b^{*} \in Q\left(x^{*}, h\left(x^{*}\right)\right), c \in F(g(y))$ and $d^{*} \in F\left(h\left(x^{*}\right)\right)$.

(ii) Since $(F, Q)$ is locally non-positive at $x_{0} \in K$ with respect to $(g, h)$, for $x^{*} \in K \cap \partial N\left(x_{0}\right)$ there exists $z_{0} \in K \cap \operatorname{Int} N\left(x_{0}\right)$ such that

$$
a_{0}-b^{*}+c_{0}-d^{*} \in-P\left(x^{*}\right)
$$

for any $a_{0} \in Q\left(x^{*}, g\left(z_{0}\right)\right), b^{*} \in Q\left(x^{*}, h\left(x^{*}\right)\right), c_{0} \in F\left(g\left(z_{0}\right)\right)$ and $d^{*} \in F\left(h\left(x^{*}\right)\right)$.

By a similar method, for any $y \in K$, there exists a $t \in(0,1)$ such that $t\left(y-z_{0}\right) \in N\left(x_{0}\right) \backslash\left\{z_{0}\right\}$, so $z_{t}:=t y+(1-t) z_{0} \in K \cap N\left(x_{0}\right)$. Hence it follows from (3.1)

$$
a_{t}-b^{*}+c_{t}-d^{*} \in P\left(x^{*}\right)
$$

for any $a_{t} \in Q\left(x^{*}, g\left(z_{t}\right)\right), b^{*} \in Q\left(x^{*}, h\left(x^{*}\right)\right), c_{t} \in F\left(g\left(z_{t}\right)\right)$ and $d^{*} \in F\left(h\left(x^{*}\right)\right)$. Letting $t \rightarrow 0$ in (3.4), we obtain

$$
a_{0}-b^{*}+c_{0}-d^{*} \in P\left(x^{*}\right)
$$

for any $a_{0} \in Q\left(x^{*}, g\left(z_{0}\right)\right), b^{*} \in Q\left(x^{*}, h\left(x^{*}\right)\right), c_{0} \in F\left(g\left(z_{0}\right)\right)$ and $d^{*} \in F\left(h\left(x^{*}\right)\right)$. Thus by (3.3) and (3.5),

$$
a_{0}-b^{*}+c_{0}-d^{*}=0
$$

for any $a_{0} \in Q\left(x^{*}, g\left(z_{0}\right)\right), b^{*} \in Q\left(x^{*}, h\left(x^{*}\right)\right), c_{0} \in F\left(g\left(z_{0}\right)\right)$ and $d^{*} \in F\left(h\left(x^{*}\right)\right)$. Thus by (3.4) and (3.6), we have

$$
t a_{t}^{*}+(1-t) b^{*}-a_{0}+t c_{t}+(1-t) d^{*}-c_{0} \in P\left(x^{*}\right)
$$

for any $a_{t}^{*} \in Q\left(x^{*}, g\left(z_{t}\right)\right), b^{*} \in Q\left(x^{*}, h\left(x^{*}\right)\right), a_{0} \in Q\left(x^{*}, g\left(z_{0}\right)\right), c_{t} \in F\left(g\left(z_{t}\right)\right)$, $d^{*} \in F\left(h\left(x^{*}\right)\right)$ and $c_{0} \in F\left(g\left(z_{0}\right)\right)$.

Hence by (3.6) and (3.7)

$$
a_{t}^{*}-b^{*}+c_{t}-d^{*} \in P\left(x^{*}\right)
$$


for any $a_{t}^{*} \in Q\left(x^{*}, g\left(z_{t}\right)\right), b^{*} \in Q\left(x^{*}, h\left(x^{*}\right)\right), c_{t} \in F\left(g\left(z_{t}\right)\right)$, and $d^{*} \in F\left(h\left(x^{*}\right)\right)$. Letting $t \rightarrow 1$ in (3.8), by the condition (d) we have

$$
a^{*}-b^{*}+c-d^{*} \in P\left(x^{*}\right)
$$

for any $a^{*} \in Q\left(x^{*}, g(y)\right), b^{*} \in Q\left(x^{*}, h\left(x^{*}\right)\right), c \in F(g(y))$ and $d^{*} \in F\left(h\left(x^{*}\right)\right)$.

Hence by (i) and (ii), the proof is completed.

Letting $D=K$ in the condition (c) of Theorem 3.1, we have the following result as a corollary.

Theorem 3.2. Let $K$ be a nonempty compact and convex subset of a real Banach space X, and assume that the condition (a), (b) and (d) of Theorem 3.1 hold with the following condition (c)' instead of (c) of Theorem 3.1;

$(\mathrm{c})^{\prime}$ the mappings $(F, Q)$ is locally non-positive at $x_{0} \in K$ with respect to $(g, h)$.

Then $(F Q-V I)$ has a solution in the neighborhood of $x_{0}$, that is, there exists $x^{*} \in K \cap N\left(x_{0}\right)$ such that, for $y \in K$

$$
a^{*}-b^{*}+c-d^{*} \in P\left(x^{*}\right)
$$

for any $a^{*} \in Q\left(x^{*}, g(y)\right), b^{*} \in Q\left(x^{*}, h\left(x^{*}\right)\right), c \in F(g(y))$ and $d^{*} \in F\left(h\left(x^{*}\right)\right)$.

Theorem 3.3. Assume that

(a) $g, h: K \rightarrow K$ are continuous and surjective, set-valued mappings $F: K \rightarrow 2^{Y}$ and $Q: K \times K \rightarrow 2^{Y}$ are continuous and $P$ is upper semicontinuous,

(b) a single-valued mapping $T: K \times K \rightarrow Y$ satisfies

(b1) for $x \in K, T(x, x) \in P(x)$,

(b2) for $x, y \in K$,

$$
a-b+c-d-T(x, y) \in P(x)
$$

for any $a \in Q(x, g(y)), b \in Q(x, h(x)), c \in F(g(y))$ and $d \in$ $F(h(x))$,

(b3) for $x \in K$ the set $\{y \in K: T(x, y) \notin P(x)\}$ is convex,

(c) $(F, Q)$ is locally non-positive at $x_{0} \in K$ with respect to $(g, h)$, and there exists a nonempty compact and convex subset $D$ of $K \cap N\left(x_{0}\right)$ such that for all $x \in K \cap N\left(x_{0}\right) \backslash D$ there exists $y \in D$ satisfying

$$
a-b+c-d \notin P(x)
$$

for any $a \in Q(x, g(y)), b \in Q(x, h(x)), c \in F(g(y))$ and $d \in F(h(x))$.

(d) $g$ and $F$ are linear and $Q$ is linear in the second argument.

Then $(F Q-C P)$ has a solution in the neighborhood of $x_{0}$, that is, there exists $x^{*} \in K \cap N\left(x_{0}\right)$ such that,

$$
a^{*}+b^{*}=0 \quad \text { for any } a^{*} \in Q\left(x^{*}, h\left(x^{*}\right)\right) \text { and } b^{*} \in F\left(h\left(x^{*}\right)\right)
$$


and for $y \in K$,

$$
a_{y}^{*}+c \in P\left(x^{*}\right) \quad \text { for any } a_{y}^{*} \in Q\left(x^{*}, g(y)\right) \text { and } c \in F(g(y)) .
$$

Proof. The conclusion follows directly from Theorem A and Theorem 3.1.

Remark 3.1. Though Theorem A is used to prove Theorem 3.3 and Theorem B is used to prove Theorem 3.1 and Theorem 3.2, Theorem 3.1, 3.2 and 3.3 extend and generalize Theorems $\mathrm{A}$ and $\mathrm{B}$.

\section{References}

[1] Y. P. Fang and N. J. Huang, The vector F-complementarity problem with demipseudomonotone mappings in Banach spaces, Appl. Math. Lett. 16 (2003), 1019-1024.

[2] F. Ferro, A minimax theorem for vector-valued functions, J. Optim, Theory Appl. 60 (1989), 19-31.

[3] N. J. Huang and J. Li, F-implicit complementarity problems in Banach spaces, Z. Anal. Anwendungen 23 (2004), 293-302.

[4] B. S. Lee, Mixed vector FQ-implicit variational inequalities with $F Q$-complementatity problems, submitted.

[5] B. S. Lee, M. F. Khan, and Salahuddin, Vector F-implicit complementarity problems with corresponding variational inequality problems, Appl. Math. Lett. 20 (2007), 433-438.

[6] J. Li and N. J. Huang, Vector F-implicit complementarity problems in Banach spaces, Appl. Math. Lett. 19 (2006), 464-471.

[7] H. Y. Yin, C. X. Xu, and Z. X. Zhang, The F-complementarity problems and its equivalence with the least element problem, Acta Math. Sinica 44 (2001), 679-686.

Department of Mathematics

KYUNGSUNG UNIVERSITY

BUSAN 608-736, Korea

E-mail address: bslee@ks.ac.kr 